\title{
Molecular line signatures of cloud-cloud collisions
}

\author{
F. D. Priestley ${ }^{\star}$ and A. P. Whitworth \\ School of Physics and Astronomy, Cardiff University, Queen's Buildings, The Parade, Cardiff CF24 3AA, UK
}

Accepted 2021 June 14. Received 2021 May 28; in original form 2021 January 22

\begin{abstract}
Collisions between interstellar gas clouds are potentially an important mechanism for triggering star formation. This is because they are able to rapidly generate large masses of dense gas. Observationally, cloud collisions are often identified in positionvelocity (PV) space through bridging features between intensity peaks, usually of CO emission. Using a combination of hydrodynamical simulations, time-dependent chemistry, and radiative transfer, we produce synthetic molecular line observations of overlapping clouds that are genuinely colliding, and overlapping clouds that are just chance superpositions. Molecules tracing denser material than $\mathrm{CO}$, such as $\mathrm{NH}_{3}$ and $\mathrm{HCN}$, reach peak intensity ratios of 0.5 and 0.2 with respect to $\mathrm{CO}$ in the "bridging feature' region of PV space for genuinely colliding clouds. For overlapping clouds that are just chance superpositions, the peak $\mathrm{NH}_{3}$ and $\mathrm{HCN}$ intensities are co-located with the CO intensity peaks. This represents a way of confirming cloud collisions observationally and distinguishing them from chance alignments of unrelated material.
\end{abstract}

Key words: astrochemistry-stars: formation-ISM: clouds - ISM: molecules-ISM: structure.

\section{INTRODUCTION}

Collisions between molecular clouds may trigger or accelerate star formation, and galactic-scale simulations suggest that cloud collisions are relatively common events (Tasker \& Tan 2009; Dobbs, Pringle \& Duarte-Cabral 2015). While the majority of these collisions may have little effect on star formation and simply act to restructure the clouds involved, if some fraction of collisions trigger gravitational collapse, then the observed link between global galaxy properties and local star formation (Kennicutt 1998) can be reproduced (Tan 2000; Fujimoto, Tasker \& Habe 2014).

Cloud collisions with relative velocities of $\sim 10 \mathrm{~km} \mathrm{~s}^{-1}$ appear to be fast enough to result in significant compression at the interface between the clouds, thereby promoting star formation, without being so fast that the clouds are entirely disrupted (Takahira, Tasker \& Habe 2014; Balfour et al. 2015; Balfour, Whitworth \& Hubber 2017; Liow \& Dobbs 2020). There are several known cases of active starforming regions associated with multiple molecular gas components separated by velocities of this order (e.g. Fukui et al. 2015; Dobashi et al. 2019), suggesting that there has been collisional triggering.

While the presence of multiple velocity components in molecular line emission may be suggestive of an ongoing cloud collision, it is not conclusive proof, and additional signatures such as cloud-scale emission from shock tracers (Jiménez-Serra et al. 2010; Cosentino et al. 2018, 2020) are necessary to distinguish collisions from chance line-of-sight alignments. The most commonly used signature is the presence of 'bridging features' in position-velocity (PV) maps of CO isotopologues (Fukui et al. 2020, and references therein). First identified in combined hydrodynamical-radiative transfer modelling of cloud collisions by Haworth et al. (2015a), bridging features result from the deceleration of material at the interface between

^E-mail: priestleyf@cardiff.ac.uk two colliding clouds. This produces a shock-compressed layer that emits at velocities intermediate between the two intensity peaks corresponding to the undecelerated clouds.

While easy to search for, bridging features are present for only a small fraction of the cloud lifetime (Haworth et al. 2015b) and become increasingly indistinct as the viewing angle approaches $90^{\circ}$ to the collision axis (Haworth et al. 2015a; Bisbas et al. 2017). Additionally, the high abundance and low critical density of $\mathrm{CO}$ result in relatively strong emission even in low-density gas. It would therefore not be surprising to find some emission between two intensity peaks in PV space, even if the peaks did not represent colliding clouds. While some proposed bridging features are remarkably similar to those in the Haworth et al. (2015a) simulations (Torii et al. 2011), others are much less clear-cut (e.g. Issac et al. 2020, although we note that those authors present a significant quantity of other evidence in favour of a cloud collision).

Previous theoretical work has focused on the $\operatorname{CO}(J=1-0)$ rotational transition, specifically the ${ }^{12} \mathrm{CO}$ isotopologue (Haworth et al. 2015a), and on atomic fine-structure lines (Bisbas et al. 2017), which predominantly trace lower density gas. At higher densities, such as those found in the interface between colliding clouds, optical depth effects and depletion on to grain surfaces result in a poor correlation between CO intensity and gas mass (e.g. Priestley \& Whitworth 2020). Moreover, the low abundance of atomic species in dense gas makes them unsuitable as tracers of shock-compressed layers. In this paper, we use combined hydrodynamical, chemical, and radiative transfer models to investigate line emission from molecules requiring higher excitation densities as potential signatures of ongoing collisions between molecular clouds.

\section{METHOD}

We perform hydrodynamical simulations using PHANTOM (Price et al. 2018), a smoothed-particle hydrodynamics (SPH) code. We 



Figure 1. Surface density maps from the cloud-collision simulation at $t_{\mathrm{end}}=0.49 \mathrm{Myr}$. Left-hand panel: looking along the collision axis, i.e. projected on the $z=0$ plane. Right-hand panel: looking perpendicular to the collision axis, i.e. projected on the $y=0$ plane.

consider a head-on collision between two uniform-density spherical clouds: a large cloud with radius $R_{\text {large }}=5 \mathrm{pc}$, mass $M_{\text {large }}=$ $10^{4} \mathrm{M}_{\odot}$, and density $\rho_{\text {large }}=1.3 \times 10^{-21} \mathrm{~g} \mathrm{~cm}^{-3}$, and a small cloud with $R_{\text {small }}=2.5 \mathrm{pc}, M_{\text {small }}=2.5 \times 10^{3} \mathrm{M}_{\odot}$, and $\rho_{\text {small }}=2.6 \times$ $10^{-21} \mathrm{~g} \mathrm{~cm}^{-3}$. Assuming a fractional hydrogen abundance by mass of $X=0.70$, the corresponding molecular hydrogen densities are $n_{\text {large }}=280 \mathrm{H}_{2} \mathrm{~cm}^{-3}$ and $n_{\text {small }}=560 \mathrm{H}_{2} \mathrm{~cm}^{-3}$. These cloud masses are somewhat smaller than the representative $\sim 10^{5} \mathrm{M}_{\odot}$ found by Dobbs et al. (2015) in galactic-scale simulations but more in line with those in previous theoretical studies of individual cloud collisions (e.g. Haworth et al. 2015a) and their role in triggering star formation (e.g. Balfour et al. 2015). We model the initial clouds with randomly positioned SPH particles, each having mass $m_{\mathrm{SPH}}=0.05 \mathrm{M}_{\odot}$. This results in a non-uniform density distribution, with fractional fluctuations $\Delta \ln (\rho) \sim 0.7$ about the mean. The gas in the clouds is isothermal, with sound speed $c_{\mathrm{S}}=0.2 \mathrm{~km} \mathrm{~s}^{-1}$, corresponding to a gas-kinetic temperature of $10 \mathrm{~K}$ if the hydrogen is molecular. In addition, we introduce a turbulent velocity field with power spectrum $P(k) \propto k^{-4}$ and root-mean-squared velocity $2.4 \mathrm{~km} \mathrm{~s}^{-1}$ (i.e. Mach 12), consistent with the observed turbulent properties of molecular clouds (Larson 1981).

At the outset, the cloud centres are at $z_{\text {large }}=+R_{\text {large }}$ and $z_{\text {small }}=$ $-R_{\text {small }}$, so that the two clouds are initially just touching. The rest of the computational domain (a cubic periodic box with side length $80 \mathrm{pc}$ ) is filled with an ambient medium having density 100 times lower, and sound speed 10 times higher, than in the cloud; this ensures approximate pressure balance across the boundary of the cloud. In the 'cloud-collision simulation', the clouds have initial velocities of $v_{\text {large }}=-5 \mathrm{~km} \mathrm{~s}^{-1}$ and $v_{\text {small }}=+5 \mathrm{~km} \mathrm{~s}^{-1}$ along the $z$-axis, giving a collision velocity of $v_{\text {col }}=10 \mathrm{~km} \mathrm{~s}^{-1}$, comparable to the more violent (and presumably more readily observable) collisions reported by Dobbs et al. (2015), and again in line with previous studies of observational cloud-collision signatures (Haworth et al. 2015a; Bisbas et al. 2017). As a consequence, a dense, shockcompressed layer forms between the clouds. This material has a range of velocities between $\sim v_{\text {large }}$ and $\sim v_{\text {small }}$ but is concentrated in the range $1.5 \mathrm{~km} \mathrm{~s}^{-1} \lesssim v \lesssim 3.5 \mathrm{~km} \mathrm{~s}^{-1}$, which is due to conservation of momentum and the fact that the material in the small cloud is twice as dense as that in the large cloud.

For the purpose of comparison, we also perform a 'chancealignment simulation', in which $v_{\text {large }}=v_{\text {small }}=v_{\text {col }}=0 \mathrm{~km} \mathrm{~s}^{-1}$, i.e. the clouds evolve independently (that is, apart from their mutual gravitational attraction). We follow the evolution of both the cloudcollision simulation and the chance-alignment simulation for a time $t_{\text {end }}=R_{\text {small }} / v_{\text {small }}=0.49 \mathrm{Myr}$. We introduce sink particles above a threshold density $\rho_{\text {sink }}=10^{-14} \mathrm{~g} \mathrm{~cm}^{-3}$, with an accretion radius $r_{\text {sink }}=0.01 \mathrm{pc}$, following the prescription in Price et al. (2018).

In the cloud-collision simulation, we track the chemical evolution of all particles within $5 \mathrm{pc}$ of the origin at $t_{\mathrm{end}}$ (and for the chancealignment simulation, within $10 \mathrm{pc}$ of the origin at $\left.t_{\mathrm{end}}\right) \mathrm{using}$ UCLCHEM (Holdship et al. 2017), a time-dependent gas-grain code, with the UMIST12 reaction network (McElroy et al. 2013) and the high-metal elemental abundances from Lee et al. (1998). We assume the standard interstellar background radiation field of Habing (1968). To account for attenuation by dust, we set the average column density shielding a gas particle to

$N_{\mathrm{H}_{2}}=n_{\mathrm{H}_{2}} c_{\mathrm{S}}(G \rho)^{-1 / 2}$,

i.e. the local density multiplied by the Jeans length, following Priestley \& Whitworth (2020). We then convert $N_{\mathrm{H}_{2}}$ to a visual extinction using $A_{\mathrm{V}}=N_{\mathrm{H}_{2}} /\left(3.3 \times 10^{21} \mathrm{~cm}^{-2}\right)$ (Bohlin, Savage \& Drake 1978).

We use the results of our chemical modelling to calculate molecular line intensities using LIME (Brinch \& Hogerheijde 2010), a line radiative transfer code. Dust optical properties are taken from Ossenkopf \& Henning (1994) and molecular data from the LAMDA database (Schöier et al. 2005). We use 50000 uniformly distributed sample points and assign to each sample point the properties of the nearest SPH particle. Increasing the number of sample points incurs a prohibitive computational overhead and does not substantially change our results. Our output position-position-velocity cubes have a spatial resolution of $0.025 \mathrm{pc}$ per pixel and cover velocities from -6 to $6 \mathrm{~km} \mathrm{~s}^{-1}$ in $0.05 \mathrm{~km} \mathrm{~s}^{-1}$ bins.

\section{RESULTS}

Fig. 1 shows the surface density of the colliding clouds at $t_{\text {end }}=$ $0.49 \mathrm{Myr}$, viewed along and perpendicular to the collision axis. From Fig. 1, we see (a) that the shock-compressed layer has a lateral extent $\Delta x \sim \Delta y \sim 4 \mathrm{pc}$, and (b) that the shocked gas will have a range of velocities, first because the unshocked gas at different distances from the collision axis hits the shock at different angles, and secondly 



Figure 2. Integrated intensity maps of the $\mathrm{CO}$ line (left-hand panels), the $\mathrm{NH}_{3}$ line (middle panels), and the HCN line (right-hand panels), from the cloudcollision simulation at $t_{\mathrm{end}}=0.49 \mathrm{Myr}$, looking along the collision axis (top row) and perpendicular to the collision axis (bottom row).

because the shocked gas expands away behind the shock. Moreover, regardless of viewing angle, the gas density is dominated by the dense material in the shock-compressed layer between the colliding clouds. This results in significant differences in the appearance of the clouds when mapped in different molecular lines, depending on the abundance of the molecule in question and the excitation conditions of the transition.

For example, Fig. 2 shows the corresponding integrated intensity maps for the ${ }^{12} \mathrm{CO}(J=1-0), \mathrm{NH}_{3}(1,1)$, and $\mathrm{HCN}(J=1-0)$ lines, ${ }^{1}$ at the same time $\left(t_{\text {end }}=0.49 \mathrm{Myr}\right)$, looking along and perpendicular to the collision axis. While there is significant ${ }^{12} \mathrm{CO}$ emission from the cloud material that has not yet been shocked, the $\mathrm{NH}_{3}$ and $\mathrm{HCN}$ lines preferentially trace denser gas in the shock-compressed layer. We note that no molecular line directly traces the underlying gas density (i.e. with integrated intensity linearly proportional to surface density).

Fig. 3 shows PV maps of the same three molecular lines (CO, $\mathrm{NH}_{3}$, and $\mathrm{HCN}$ ). The line intensities have been averaged along the $y$ axis to compensate for the limited resolution of our LIME models and to improve the signal to noise. Viewed along the collision axis, the $\mathrm{CO}$ emission shows the bridging feature identified by Haworth et al. (2015a) for the cloud-collision simulation; both the large and small clouds are visible as peaks in the line intensity, separated in velocity space, with weaker emission at velocities between the two peaks, due to the gas in the shock-compressed layer. Molecules tracing denser gas than CO have very different PV morphologies. The large cloud is invisible in both $\mathrm{NH}_{3}$ and $\mathrm{HCN}$ emission, and even the small cloud (with its higher density) is barely visible in HCN. Only the dense gas in the shock-compressed layer shows substantial emission from $\mathrm{NH}_{3}$ and $\mathrm{HCN}$.

Fig. 3 also shows PV maps of the $\mathrm{CO}, \mathrm{NH}_{3}$, and $\mathrm{HCN}$ lines for the chance-alignment simulation, seen at the same time $\left(t_{\text {end }}=\right.$

${ }^{1}$ In the following, we will refer to these lines simply as the $\mathrm{CO}, \mathrm{NH}_{3}$, and HCN lines.
0.49 Myr) and along the same axis as the cloud-collision simulation, so that the two clouds are aligned one behind the other. As in the cloud-collision simulation, the $\mathrm{NH}_{3}$ and $\mathrm{HCN}$ intensity peaks still highlight the denser regions of the clouds, preferentially located towards the cloud centres. However, unlike the cloud-collision simulation, the ranges of velocity for the $\mathrm{NH}_{3}$ and $\mathrm{HCN}$ lines are essentially the same as for the $\mathrm{CO}$ line. This is because the $\mathrm{NH}_{3}$ and $\mathrm{HCN}$ emission is due to denser than average turbulent elements in the clouds that have essentially the same velocity dispersion as the more widely distributed CO-emitting gas. We therefore suggest that this comparative analysis of line intensities from molecules with different characteristic densities (Shirley 2015) constitutes an additional observational signature of an ongoing cloud collision.

This is even more clearly visible in PV maps of the line intensity ratios, as shown in Fig. 4. While the ratio of $\mathrm{NH}_{3}$ emission to $\mathrm{CO}$ emission is $<0.1$ in the large cloud and $\sim 0.3$ in the denser small cloud in the cloud-collision simulation, it reaches values of $\sim 0.5$ at the intermediate velocities of the shock-compressed layer. Similarly, the ratio of $\mathrm{HCN}$ emission to $\mathrm{CO}$ emission is $<0.02$ in the large cloud and $\sim 0.06$ in the small cloud but reaches values $\sim 0.15$ at the intermediate velocities of the shock-compressed layer. While the chance-alignment simulation shows comparable maximum values of the intensity ratios for both molecules, these are not localized in a particular region of PV space but occur over the same range as the $\mathrm{CO}$ emission.

PV maps of the line intensities and intensity ratios for the collidingcloud simulation at the earlier time of $t_{\text {end }} / 2 \sim 0.24 \mathrm{Myr}$ are shown in Figs 3 and 4, respectively. At this point, the $\mathrm{CO}$ bridging feature is relatively indistinct, but the enhancement in emission from $\mathrm{NH}_{3}$ and $\mathrm{HCN}$ is still apparent. The strength of the signature, both in absolute terms and relative to the CO intensity, is reduced but is still clearly distinct from the chance-alignment simulation, where intensity peaks of CO and denser gas tracers are closely correlated.

Some elements of the signatures explored above still obtain, even when the cloud-collision simulation is viewed perpendicular to the 

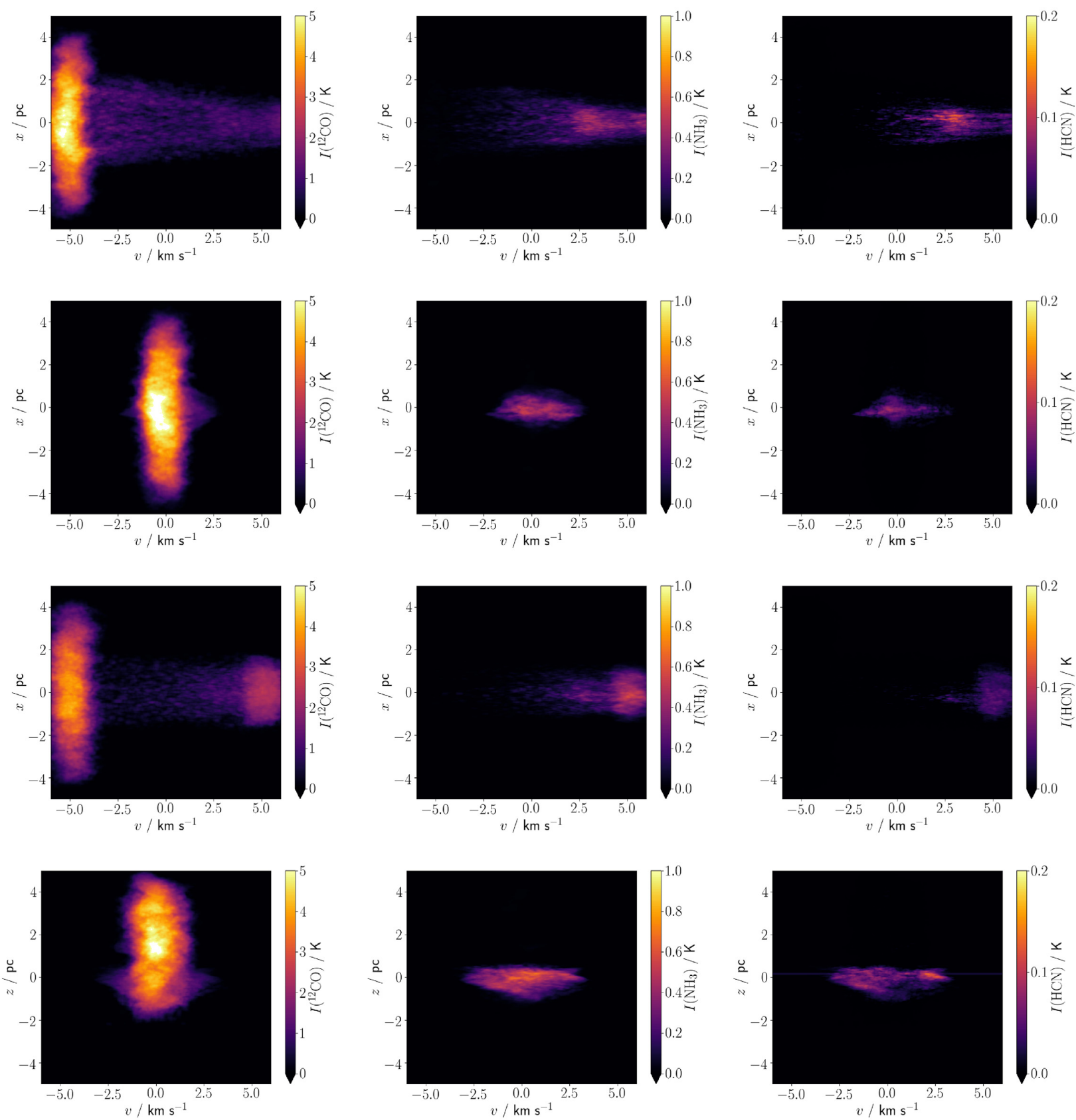

Figure 3. Position-velocity intensity maps of the $\mathrm{CO}$ line (left-hand panels), the $\mathrm{NH}_{3}$ line (middle panels), and the HCN line (right-hand panels). First row: the cloud-collision simulation at $t_{\mathrm{end}}=0.49 \mathrm{Myr}$, looking along the collision axis. Second row: the chance-alignment simulation at $t_{\mathrm{end}}=0.49 \mathrm{Myr}$, looking along the collision axis. Third row: the cloud-collision simulation at $t_{\mathrm{end}}=0.24 \mathrm{Myr}$, looking along the collision axis. Fourth row: the cloud-collision simulation at $t_{\text {end }}=0.49 \mathrm{Myr}$, looking perpendicular to the collision axis. The intensities have been averaged along the $y$-axis to improve the signal to noise.

collision axis. The integrated line intensities from the cloud-collision simulation at $t_{\text {end }}=0.49 \mathrm{Myr}$, viewed perpendicular to the collision axis, are shown in Fig. 2. The compressed layer is highlighted in all three molecules but particularly in those tracing denser gas $\left(\mathrm{NH}_{3}\right.$ and $\mathrm{HCN}$ ). It may therefore sometimes be possible to trace the bow shock where a denser smaller cloud is ploughing into a more rarefied larger cloud. However, in this situation, there is essentially no velocity information to support the identification of a cloud collision.

Fig. 3 shows $\mathrm{PV}$ maps of the $\mathrm{CO}, \mathrm{NH}_{3}$, and $\mathrm{HCN}$ lines from the cloud-collision simulation at $t_{\text {end }}=0.49 \mathrm{Myr}$, where the position $(\mathrm{P})$ axis is the collision axis, i.e. the $z$-axis. In this case, the observer is looking along the $y$-axis, and the intensities have been averaged along the $x$-axis to improve the signal to noise. The velocity range for the $\mathrm{NH}_{3}$ and $\mathrm{HCN}$ emission from the cloud-collision simulation viewed perpendicular to the collision axis is a little larger than for the chance-alignment simulation, but this systematic difference only obtains here because the individual clouds involved are identical apart from their initial bulk velocities. The $z$ range of the $\mathrm{NH}_{3}$ and $\mathrm{HCN}$ emission relative to the $z$ range of the $\mathrm{CO}$ emission cannot be used to distinguish the cloud-collision simulation from the chancealignment simulation: for the cloud-collision simulation, the relative $z$ ranges depend on the relative masses and densities of the two clouds 



Figure 4. Position-velocity intensity-ratio maps of the $\mathrm{NH}_{3}$ line (left-hand panels) and the $\mathrm{HCN}$ line (right-hand panels), both relative to the CO line. First row: the cloud-collision simulation at $t_{\mathrm{end}}=0.49 \mathrm{Myr}$, looking along the collision axis. Second row: the chance-alignment simulation at $t_{\mathrm{end}}=0.49 \mathrm{Myr}$, looking along the collision axis. Third row: the cloud-collision simulation at $t_{\mathrm{end}}=0.24 \mathrm{Myr}$, looking along the collision axis. The intensities have been averaged along the $y$-axis to improve the signal to noise.

involved in the collision; for the chance-alignment simulation, the relative $z$ ranges depend on the positions of the two clouds (which are not necessarily exactly aligned relative to the observer, as we have assumed here).

\section{DISCUSSION AND CONCLUSIONS}

Since the enhanced dense gas emission from $\mathrm{NH}_{3}$ and $\mathrm{HCN}$ occurs in the same region of $\mathrm{PV}$ space as the $\mathrm{CO}$ bridging feature, identifying this enhanced dense gas emission requires that a bridging feature is 
visible in the first place. Enhanced dense gas emission is therefore limited - as a cloud-collision signature - by the same issues regarding the lifetimes of bridging features, and their viewing angle dependency, as discussed in Haworth et al. (2015b) and Bisbas et al. (2017). However, while the atomic fine-structure lines investigated in Bisbas et al. (2017) trace essentially the same material as the CO line, molecular lines like $\mathrm{NH}_{3}$ and $\mathrm{HCN}$, which require higher excitation densities, are necessarily strongly enhanced in the compressed layer resulting from a cloud collision. $\mathrm{NH}_{3}$ data are available for many Galactic molecular clouds (e.g. Friesen et al. 2017), and lines from other dense gas tracers can be observed simultaneously with $\mathrm{CO}$, using sub-millimetre telescopes. Our proposed collision signature should thus be relatively simple to investigate in observational studies.

To summarize, we have performed combined hydrodynamical, chemical, and radiative-transfer simulations of both colliding clouds and isolated but aligned clouds, in order to evaluate molecular line signatures of the underlying dynamics. We find that molecules tracing dense gas, such as $\mathrm{NH}_{3}$ and $\mathrm{HCN}$, have strongly enhanced emission in the shock-compressed layer between colliding clouds and therefore serve to highlight CO 'bridging features'. This can be used to confirm that observed bridging features are due to colliding clouds, rather than unrelated clouds along the line of sight.

\section{ACKNOWLEDGEMENTS}

We thank the referee for a constructive report that significantly improved this paper. We are grateful to Giuliana Cosentino and Tom Haworth for their considerate responses to persistent questioning. FDP and APW acknowledge the support of a consolidated grant (ST/K00926/1) from the UK Science and Technology Facilities Council (STFC).

\section{DATA AVAILABILITY}

The data underlying this article will be shared on request. All software used is publically available.

\section{REFERENCES}

Balfour S. K., Whitworth A. P., Hubber D. A., Jaffa S. E., 2015, MNRAS, 453,2471
Balfour S. K., Whitworth A. P., Hubber D. A., 2017, MNRAS, 465, 3483

Bisbas T. G., Tanaka K. E. I., Tan J. C., Wu B., Nakamura F., 2017, ApJ, 850, 23

Bohlin R. C., Savage B. D., Drake J. F., 1978, ApJ, 224, 132

Brinch C., Hogerheijde M. R., 2010, A\&A, 523, A25

Cosentino G. et al., 2018, MNRAS, 474, 3760

Cosentino G. et al., 2020, MNRAS, 499, 1666

Dobashi K., Shimoikura T., Katakura S., Nakamura F., Shimajiri Y., 2019, PASJ, 71, S12

Dobbs C. L., Pringle J. E., Duarte-Cabral A., 2015, MNRAS, 446, 3608

Friesen R. K. et al., 2017, ApJ, 843, 63

Fujimoto Y., Tasker E. J., Habe A., 2014, MNRAS, 445, L65

Fukui Y. et al., 2015, ApJ, 807, L4

Fukui Y., Habe A., Inoue T., Enokiya R., Tachihara K., 2020, PASJ, 73, S1

Habing H. J., 1968, Bull. Astron. Inst. Netherlands, 19, 421

Haworth T. J. et al., 2015a, MNRAS, 450, 10

Haworth T. J., Shima K., Tasker E. J., Fukui Y., Torii K., Dale J. E., Takahira K., Habe A., 2015b, MNRAS, 454, 1634

Holdship J., Viti S., Jiménez-Serra I., Makrymallis A., Priestley F., 2017, AJ, 154,38

Issac N., Tej A., Liu T., Wu Y., 2020, MNRAS, 499, 3620

Jiménez-Serra I., Caselli P., Tan J. C., Hernand ez A. K., Fontani F., Butler M. J., van Loo S., 2010, MNRAS, 406, 187

Kennicutt Robert C. J., 1998, ApJ, 498, 541

Larson R. B., 1981, MNRAS, 194, 809

Lee H.-H., Roueff E., Pineau des Forets G., Shalabiea O. M., Terzieva R., Herbst E., 1998, A\&A, 334, 1047

Liow K. Y., Dobbs C. L., 2020, MNRAS, 499, 1099

McElroy D., Walsh C., Markwick A. J., Cordiner M. A., Smith K., Millar T. J., 2013, A\&A, 550, A36

Ossenkopf V., Henning T., 1994, A\&A, 291, 943

Price D. J. et al., 2018, PASA, 35, e031

Priestley F. D., Whitworth A. P., 2020, MNRAS, 499, 3728

Schöier F. L., van der Tak F. F. S., van Dishoeck E. F., Black J. H., 2005, A\&A, 432, 369

Shirley Y. L., 2015, PASP, 127, 299

Takahira K., Tasker E. J., Habe A., 2014, ApJ, 792, 63

Tan J. C., 2000, ApJ, 536, 173

Tasker E. J., Tan J. C., 2009, ApJ, 700, 358

Torii K. et al., 2011, ApJ, 738, 46

This paper has been typeset from a $\mathrm{T}_{\mathrm{E}} \mathrm{X} / \mathrm{LT} \mathrm{E} \mathrm{X}$ file prepared by the author. 